\title{
Correction to: A TCAD Approach to Analyze the Performance of Dual Gate Dielectric Modulated Vertical Tunnel Field Effect Transistor Based Biosensor
}

\author{
B. Dewan ${ }^{1}$, S. Chaudhary ${ }^{1}$, N. Bohra ${ }^{2}$, A. Kumari ${ }^{2}$ and M. Yadav ${ }^{1}$ \\ ${ }^{1}$ Malaviya National Institute of Technology (MNIT), Jaipur, India \\ ${ }^{2}$ Maharaja Surajmal Institute of Technology, Delhi, India
}

Accepted: 09 February 2022 / Published online: 7 March 2022

(C) Metrology Society of India 2022

Correction to: MAPAN-Journal of Metrology Society of India https://doi.org/10.1007/s12647-021-00525-2

The authors would like to correct errors in the original publication of the article. The correct affiliation for the coauthors Navdeep Bohra and Ashish Kumari is:
Maharaja Surajmal Institute of Technology, Delhi, India.

Publisher's Note Springer Nature remains neutral with regard to jurisdictional claims in published maps and institutional affiliations.

The original article can be found online at https://doi.org/10.1007/s12647-021-00525-2.

*Corresponding author, E-mail: 2019rec9012@mnit.ac.in 